\title{
The Ki67 index a prognostic marker in medullary thyroid carcinoma
}

\author{
LE Tisell*, I,4 A Oden ${ }^{3}$, A Muth ${ }^{2}$, G Altiparmak', J Mõlne', H Ahlman' and O Nilsson ${ }^{2}$ \\ 'The Lundberg Laboratory for Cancer Research, Department of Surgery, Göteborg University Hospital, Göteborg University, Gothenburg, Sweden; \\ ${ }^{2}$ Department of Pathology, Göteborg University Hospital, Göteborg University, Gothenburg, Sweden; ${ }^{3}$ Department of Mathematics and Statistics, \\ Göteborg University Hospital, Göteborg University, Gothenburg, Sweden
}

\begin{abstract}
Our objective was to examine the usefulness of the Ki67 proliferation index as a prognostic marker in patients with medullary thyroid carcinoma (MTC). It is difficult to predict the prognosis of MTC by using conventional prognostic factors. Immunocytochemical analysis of tumour proliferation has been used as a prognostic tool in some tumours, but only rarely in MTC. In all, 7I tumours from 36 patients were investigated, by using a semiautomatic image analysis programme. On average 10000 nuclear profiles were counted per tumour, and the percentage of tumour cells expressing the proliferation marker, Ki67, was calculated. Primary tumours that had metastasised had higher Ki67 indices than primary tumours that had not metastasised. Recurrent lymph node metastasis had higher Ki67 indices than the primary tumours. By using a Poisson model, it was possible to estimate the median survival time for individual patients if the Ki67 index for the primary tumour and the age at surgery were known. The higher the Ki67 index and the age at operation were, the shorter was the survival. Estimating the median survival of individual patients will be of help for planning the patients' life and postoperative follow-up and treatment. British Journal of Cancer (2003) 89, 2093-2097. doi:I0.I038/sj.bjc.660I453 www.bjcancer.com (c) 2003 Cancer Research UK
\end{abstract}

Keywords: MTC; proliferation index; survival

Medullary thyroid carcinoma (MTC) is derived from the calcitoninproducing $\mathrm{C}$ cells of the thyroid. Determination of serum calcitonin concentrations is used to diagnose and follow patients with MTC (Wells et al, 1978; Tisell et al, 1996). In the TNM classification of thyroid tumours, papillary carcinoma with lymph node metastasis in young patients is classified as a stage I disease. A similar extent of MTC has a worse impact on survival and is classified as a stage III disease (The Committee on TNM classification UICC, 1992). Medullary thyroid carcinoma patients with lymph node metastasis often have persistent disease after the operation. In one-third of such patients, meticulous neck dissection led to normalisation of the serum calcitonin concentrations (Tisell et al, 1986; Moley et al, 1993). The prognosis of MTC patients is difficult to predict using conventional prognostic factors, for example, age, sex, stage, histopathological growth pattern, ploidy or biochemical tumour markers (Tisell et al, 1996). Half of the MTC tumours can be localised with octreotide scintigraphy due to their expression of somatostatin receptors. The visualised tumours grew more rapidly and aggressively than nonvisualised lesions, suggesting that octreotide scintigraphy may be used as a prognostic tool (Tisell et al,1997). Immunocytochemical analysis of tumour proliferation has become an important tool for the assessment of tumour aggressiveness and prognosis (Tubiana and Courdi, 1989). The aim of the present study was to evaluate tumour proliferation, measured

\footnotetext{
*Correspondence:Dr L-E Tisell; E-mail: larstisell@hotmail.com

${ }^{4}$ Dr L-E Tisell's current address: Department of Surgery, Sahlgrenska University Hospital, Blå stråket 7, Göteberg SE-4I345, Sweden Received I 8 June 2003; revised IO September 2003; accepted 6 October 2003
}

by Ki67, as a prognostic marker for MTC. For this, Ki67 indices of primary tumours were related to the clinical course of the MTC patients. The possible presence of mutation of the p53 gene was also investigated.

\section{MATERIAL AND METHODS}

\section{Patients}

Paraffin blocks with tumour tissue from 36 patients, 20 females and 16 males were retrieved. Each patient had at least one operation for MTC at the Sahlgrenska University Hospital. In all, 23 patients had sporadic MTC, 12 had MEN 2A and one a MEN 2B syndrome. The mean age (s.d.) at operation was 45 (15) years (median 44years). The tumours were classified according to the TNM classification (The Committee on TNM classification UICC, 1992).Tumour stage at initial operation was: stage I, two patients; stage II, six patients; stage III, 22 patients; and stage IV, six patients. The follow-up ended in March 2000. At diagnosis, six patients had distant metastasis. During follow-up 11 additional patients got distant metastasis. In all, 13 of the patients with distant metastasis died of MTC at a mean (s.d.) of 8 (7) years (median 7 years) after diagnosis, two patients died of unrelated causes after 17 years. A total of 21 patients were alive at follow-up at a mean of 20 (9) years (median 18 years) after the operation. All survivors were asymptomatic, but four had distant metastasis. The postoperative observation time for the total series was 15 (10) years (median 15 years). Individual clinical features of the 36 patients can be found in Table 1 .

In all, 71 tumour specimens were investigated, 36 primary tumours, 21 primary lymph node metastasis and 14 recurrent lymph node metastasis. The mean (s.d.) (median) of the Ki67 indices for all 
Table I Clinical features of the 36 MTC patients

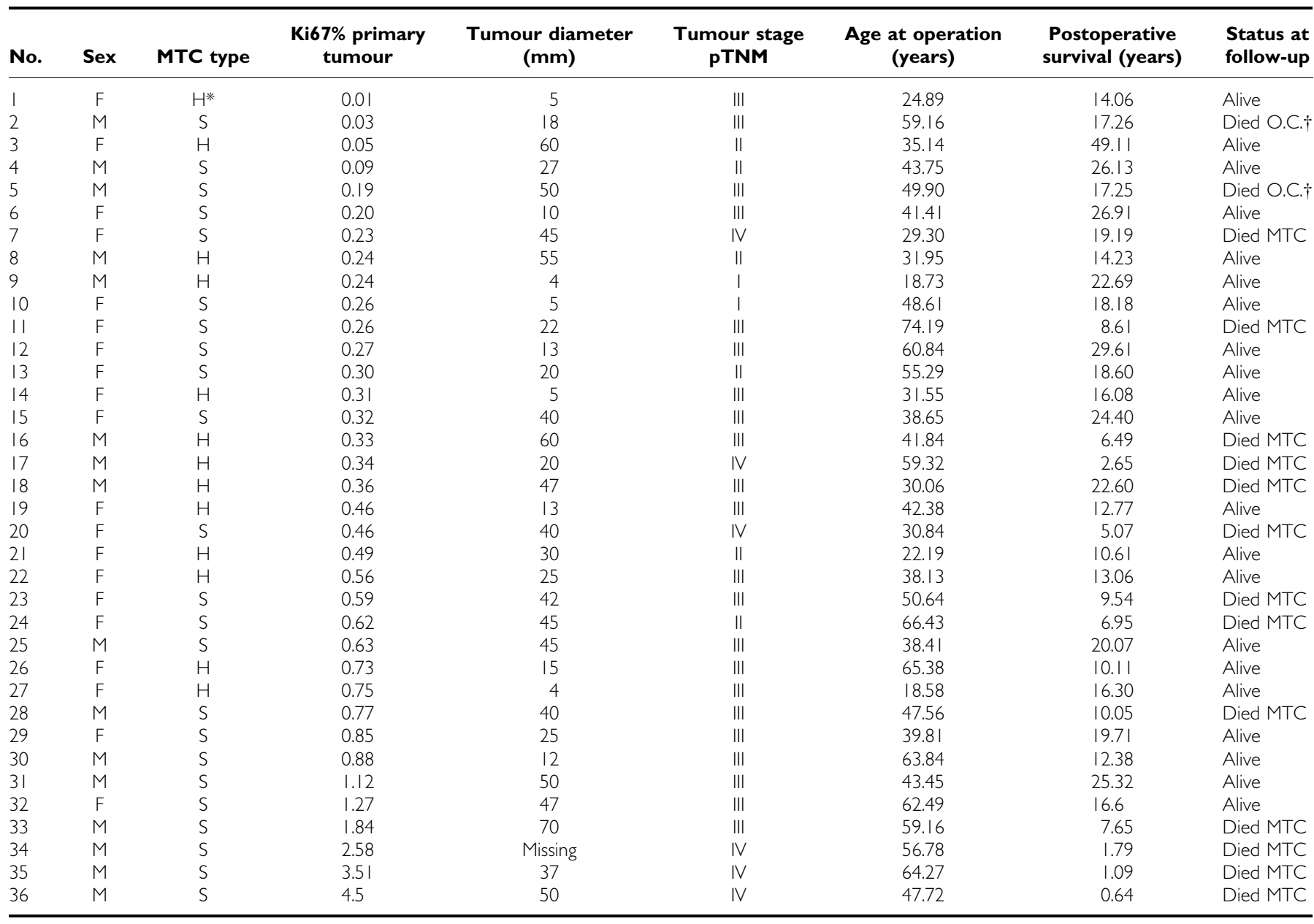

$\mathrm{H}=$ hereditary; MTC, $\mathrm{S}=$ sporadic MTC; died O.C. ${ }^{\dagger}=$ died of other causes than MTC; PTNM = postsurgical histopathological classsification; $\mathrm{T}=$ tumour; $\mathrm{N}=$ regional lymph node metastasis; $m=$ distant metastasis.

the 71 tumours was $1.71 \%$ (3.3) (median $0.61 \%$ ), the Ki67 indices of the primary tumours, primary metastasis and recurrent metastasis were $0.74 \%(0.96)$ (median $0.41 \%), 1.35 \%$ (1.49) (median $0.85 \%)$ and $4.73 \%$ (6.39) (median 1.65\%), respectively. In 12 cases, tumour tissue was available from primary tumours, primary metastasis and also from recurrent metastasis. This subset of patients was tested for within-subject differences between the Ki67 indices of the three kinds of tumours. In another analysis, the Ki67 values of the 28 primary tumours that had metastasised were compared to those of the eight tumours that had not metastasised.

\section{Therapy}

Since 1972, the operation involved a total thyroidectomy and a meticulous central lymph node dissection. Lateral lymph node dissections, on one or both sides were carried out in cases with palpable primary tumours or palpable lymph nodes in the central or lateral neck. Patients cared for at our hospital, after less radical operations performed before 1972, or after incomplete surgery elsewhere, had completion thyroidectomies with central lymph node dissections. In case of tumour recurrence repeat neck operations were performed. After the operations, the patients were followed annually at our outpatient clinic. Patients with terminal disease were followed more often. The close surveillance of the patients guaranteed that the causes of death could be verified in all cases.

\section{Immunocytochemistry}

Paraffin blocks of tumour tissue were retrieved from the Department of Pathology, Sahlgrenska University Hospital. The tissues had been routinely fixed in neutral-buffered formalin for $1-2$ days and embedded in paraffin wax. Sections $(3-4 \mu \mathrm{m})$ from primary tumours and lymph node metastasis were placed on positively charged glass slides, deparaffinised and rehydrated. Antigens were retrieved by microwave treatment in citrate buffer (10 mM citrate, $\mathrm{pH} 6.0$ ) twice for $5 \mathrm{~min}$. In order to assure even staining of tissue sections, the incubation of sections was performed in a Techmate 500 immunostainer under identical conditions. Incubation with primary antibodies was carried out for $25 \mathrm{~min}$, followed by biotinylated goat anti-mouse antibodies (LSAB, Dako), streptavidin-HRP and diaminobenzidine. After counter staining in Mayer's haematoxylin, sections were dehydrated and mounted. All antisera were diluted in antibody diluent (Tris-buffer $\mathrm{pH} 7.2$, containing $15 \mathrm{~mm}$ sodium azide and protein; Dako). The following primary antibodies were used: mouse anti-Ki67 monoclonal antibody (clone MIB-1; Immunotech) and mouse anti-p53 monoclonal antibodies (clone PAb1801 and DO-7; Dako). As negative controls served sections incubated identically, except for the primary antibody that was replaced by normal mouse IgG. As positive controls served sections of normal tissues (lymph node for Ki67) or tumour tissues (colorectal cancer for p53). 


\section{Determination of proliferation indices}

In order to estimate the growth rate of tumours, the percentage of tumour cells expressing the proliferation marker Ki67 was measured. A proliferation index was calculated for each tumour lesion by counting the total number of tumour cell nuclear profiles and the number of Ki67-positive nuclear profiles in randomly and systematically selected fields. The first field in each tumour lesion was selected randomly, and the following fields were sampled systematically using a mesh. On average 10000 nuclear profiles were counted per tumour lesion. Counting was performed using a semiautomatic image analysis programme (IBAS).

\section{Statistics}

The Mann-Whitney test was used to compare the Ki67 values of the primary tumours that had not metastasised (stages I and II) with those that had metastasised (stages III and IV). The Friedman test followed by Dunn's multiple comparison post-test was used to investigate within-subject differences between Ki67 values of primary tumours and primary and recurrent metastasis of the 12 patients who had tumour tissue available from all the three kinds of tumour lesions.

Survival analysis For the survival analysis, the following variables were noted for each patient: Ki67 index of the primary tumour, age at operation, survival time after the operation, death of MTC or not (Table 1). A Poisson model (Breslow and Day, 1987) was applied to estimate the hazard function of death from MTC. The hazard function was of the form $\exp \left(\beta_{0}+\beta_{1} \times\right.$ time since operation $+\beta_{2} \times$ current age $+\beta_{3} \times$ Ki67) Also a model including interaction between time and Ki67 was studied. For the derivation of the cause-specific median survival time see the addendum. If the age at operation and the Ki67 index are known, the formula of the last line of the addendum can be used to calculate the median survivals for individual patients.

In a Poisson regression, the variables tumour size, sex, type of tumour and tumour stage were included one at a time together with the variables Ki67 and age to find out if any of the four first mentioned variables had any significant importance for death of MTC. For these calculations, tumour size was represented by the largest tumour diameter, type of tumour was sporadic or hereditary tumours, stage was stages I and II or stage III and IV. The variables for each patient can be found in Table 1 . The two patients who died of unrelated causes were censored at the date of death and the patients alive at the end of the study were censored at that time.

\section{RESULTS}

The subset of 12 patients who had tumour tissue available from primary tumours, primary metastasis and recurrent metastasis could be used to test for within-subject differences between the three kinds of tumour lesions. In this subset of patients, the Ki67 values of the primary tumours, the primary metastasis and the recurrent metastasis were $0.80 \%(0.74)$ (median $0.53 \%), 1.56 \%$ (1.72) (median $1.11 \%$ ) and $4.62 \%$ (6.40) (median $1.65 \%$ ), respectively. The higher value for the recurrent metastasis $v s$ the primary tumours was significant $(P<0.01)$.

Primary tumours that had metastasised, stages III and IV, had higher Ki67 indices, $0.87 \%$ (1.05) $(0.51 \%)$ than primary tumours without metastasis, stages I and II, $0.29 \%(0.19)(0.25 \%)(P<0.05)$.

\section{Survival analysis}

The $\beta$-coefficients determining the hazard function and the median postoperative survival time are found in Table 2. The estimated median cause-specific survival times, at three ages, for five different Ki67 values, are presented in Table 3 . This table shows that the estimated median survival time will be reduced with higher ages at surgery and with higher Ki67 indices. Median survival times for other values of age and Ki67 can be calculated by using the formula on the last line of the addendum.

Figure 1 shows an example of three calculated curves of median survival, where the age at operation is 45 years. In contrast to the curves of the Poisson regressions is the curve of the Cox regression a step function, which is a weakness when applied to individual patients. The variables entered in the three regression curves were original data from the studied population.

When including the variables sex, tumour size, tumour type and tumour stage, one at a time together with $\mathrm{Ki} 67$ and age, in a Poisson regression none of the four first mentioned variables had any significant importance for death of tumour. Closest to significance was size with $P=0.1710$. The calculation of median survival depending on Ki67 was not affected by the new variables.

\section{p53 labelling}

Immunostaining with two different antibodies against p53 failed to label any of the investigated tumours, while control tissues (colorectal carcinoma) were positive. This is in accordance with previous studies showing the absence of p53 mutations in MTC (Yana et al, 1992; Herfarth et al, 1997).

\section{DISCUSSION}

Several studies have investigated the usefulness of proliferation markers as prognostic tools in human neoplasm, for example, softtissue tumours, prostate, ovarian and lung carcinoma (Raymond et al, 1988; Pence et al, 1993; Drobnjak et al, 1994; Kerns et al, 1994). Immunocytochemical detection of the nuclear proliferation antigen Ki67 with MIB-1 monoclonal antibody can be used to

Table $2 \beta$-coefficients for calculating the hazard function and the estimated median survival time

\begin{tabular}{lccc}
\hline Variable & $\boldsymbol{\beta}$-coefficients & s.e. & $\begin{array}{c}\text { Two-tailed } \\
\boldsymbol{P} \text {-value }\end{array}$ \\
\hline Constant & -5.9290 & 1.3405 & \\
Time since operation (years) & -0.0199 & 0.0436 & $P>0.30$ \\
Current age (years) & 0.0285 & 0.0224 & $P=0.22$ \\
Ki67\% & 1.1347 & 0.2437 & $P<0.001$ \\
\hline
\end{tabular}

Table 3 The estimated postoperative median survival for five Ki67 values at three ages

\begin{tabular}{lcc}
\hline $\begin{array}{l}\text { Ki67 index of primary } \\
\text { MTC tumours }\end{array}$ & $\begin{array}{c}\text { Age at operation } \\
\text { (years) }\end{array}$ & $\begin{array}{c}\text { Cause-specific, median } \\
\text { survival (years) }\end{array}$ \\
\hline 0.6 & 35 & 40.6 \\
0.7 & 35 & 36.8 \\
1.0 & 35 & 27.4 \\
2.0 & 35 & 9.5 \\
4.5 & 35 & 0.6 \\
& & \\
0.6 & 45 & 31.8 \\
0.7 & 45 & 28.2 \\
1.0 & 45 & 21.2 \\
2.0 & 45 & 7.2 \\
4.5 & 45 & 0.4 \\
& & \\
0.6 & 55 & 24.7 \\
0.7 & 55 & 22.2 \\
1.0 & 55 & 16.3 \\
2.0 & 55 & 5.5 \\
4.5 & 55 & 0.3 \\
\hline
\end{tabular}

Note that the higher the Ki67 index of the primary tumour and age at operation were, the shorter was the estimated postoperative survival. 


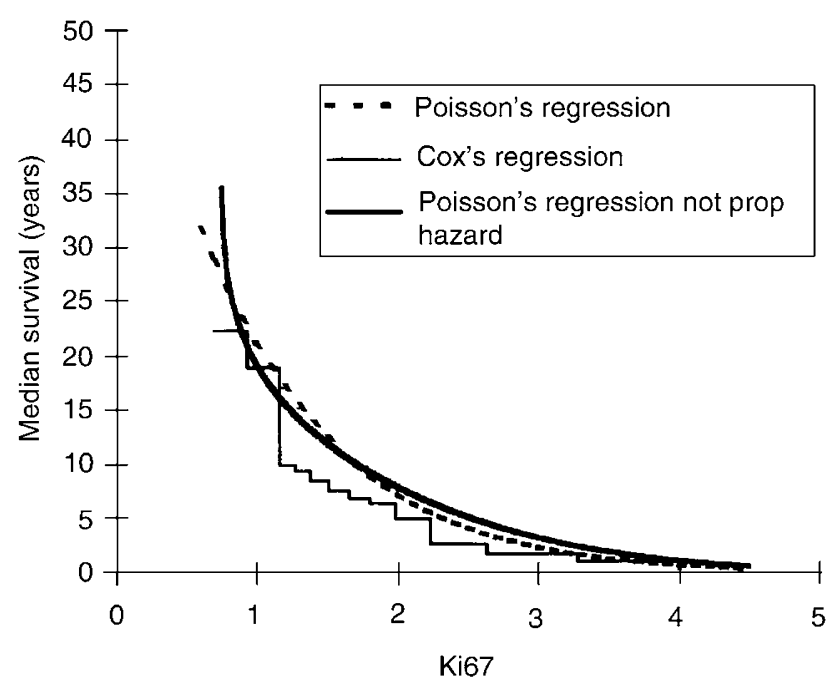

Figure I The figure shows as an example, the calculated curves of median survival where the age at operation is 45 years. The three curves show similar relations between the median survival and the Ki67 index. The curve of the Cox regression is a step function and from the figure it is apparent that this is a weakness when it is applied to individual patients. The Poisson regression with nonproportional hazard, which includes interaction between Ki67 index and time, does not differ significantly from the simpler Poisson model. By use of the Poisson regression result, the median survival of individual patients can be calculated. The variables entered in the three regression curves were original data from the studied population.

study proliferation in paraffin-embedded tumour material. This method has been used also in endocrine tumours. For thyroid tumours, it has been shown that anaplastic tumours have higher Ki67 indices than well-differentiated thyroid tumours (Carr et al, 1993), and that Hürthle cell carcinomas of the thyroid have higher Ki67 indices than benign Hürthle cell adenomas (Erickson et al, 2000). There are few studies about Ki67 indices in MTC, and these studies include few patients (Rigaud and Bogomoletz, 1991; Carr et al, 1993; Wang et al, 1996). In the present long-term follow-up study, we examined MTC tumour specimens from 36 patients to find out if the level of Ki67 expression was related to the level of malignancy. According to the TNM system, MTC tumours without metastasis are classified as stages I and II disease, while tumours with metastasis are classified as stages III and IV disease. Significantly higher Ki67 indices were found in primary tumours that had metastasised than in primary tumours without metastasis. It was also found that recurrent metastasis had higher Ki67 indices than primary tumours, which is in agreement with the observation that in papillary thyroid cancer the proportion of proliferating cells increased with each recurrence (Ozaki et al, 1999).

According to DeLellis (1995), the Ki67 index cannot be used to judge the malignancy of individual endocrine tumours, but this marker may be helpful in identifying subsets of tumours that will follow an aggressive course. To explore whether the Ki67 index could predict the cause-specific postoperative survival in individual patients, we used a Poisson model to analyse the data (Breslow and Day, 1987) and a formula was derived that can be used to estimate the cause-specific median survival time for individual patients, if the Ki67 index and age at surgery are known. Estimated individual survival times were found to be reduced with higher Ki67 indices and higher age at surgery. With the Poisson model, the estimated median survival can be obtained for every age and for every value of the Ki67 index. The median survival can also be calculated with the help of a Cox model, but compared to the Poisson model it is less appropriate since the resulting function of the Cox model is a step function, which cannot be used to obtain the median survival for every value of the Ki67 index (cf Figure 1). When including the variables tumour size, sex, type of tumour (sporadic or hereditary) and tumour stage, one at a time together with Ki67 and age, in a Poisson regression none of the four first mentioned variables had any significant importance for death of MTC. The calculation of median survival depending on Ki67 was not affected by the new variables. This shows that the Ki67 index is a very suitable prognostic marker for MTC.

Estimating the median survival of individual patients will be of help for planning the patients' life and postoperative follow-up and treatment.

\section{ADDENDUM}

Derivation of the median survival time $T$.

Let $h(t)$ be the hazard function of death.

Then $\exp \left(-\int_{0}^{T} h(t) \mathrm{d} t=0.5\right.$
Thus $\int_{0}^{T} h(t) \mathrm{d} t=-\log (0.5)$.

Let age $_{0}$ be the age at operation. Then the current age $t$ years after operation is age $e_{0}+t$.

Hence, the above equality can be written:

$$
\int_{0}^{T} \exp \left(\beta_{0}+\beta_{1} t+\beta_{2}\left(a g e_{0}+t\right)+\beta_{3} K i 67\right) d t=-\log (0.5) .
$$
Then we have $\int_{0}^{T} \exp \left(\left(\beta_{1}+\beta_{2}\right) t\right) \mathrm{d} t=-\log (0.5) \cdot \exp \left(-\left(\beta_{0}+\beta_{2}\right.\right.$
age $_{0}+\beta_{3}$ Ki67 $\left.)\right)$.

Then the integral can be calculated and thus

$$
\begin{aligned}
\left(\exp \left(\left(\beta_{1}+\beta_{2}\right) T\right)-1\right) /\left(\beta_{1}+\beta_{2}\right)= & -\log (0.5) \cdot \exp \left(-\left(\beta_{0}+\beta_{2} \text { age }_{0}\right.\right. \\
& \left.\left.+\beta_{3} \text { Ki67 }\right)\right) .
\end{aligned}
$$

Finally, $\quad T=\log \left[\left(-\log (0.5)-\exp \left(-\left(\beta_{0}+\beta_{2}\right.\right.\right.\right.$ age $e_{0}+\beta_{3}$ Ki67 $\left.)\right)$. $\left.\left.\left(\beta_{1}+\beta_{2}\right)\right)+1\right] /\left(\beta_{1}+\beta_{2}\right)$.

\section{ACKNOWLEDGEMENTS}

This study was supported by The Swedish Cancer Society (3911), The Swedish MRC (5220), IB and A Lundberg Research Foundation, Assar Gabrielsson Foundation, The Gõteborg Medical Society, The King Gustav V Jubilee Clinic Cancer Fund, Gõteborg and Sahlgrenska Hospital Research Funds.

\section{REFERENCES}

Breslow NE, Day NE (1987) IARC Scientific Publications No 32 Lyon. Statist Methods Cancer Res 2: $131-135$

Carr K, Heffes C, Jin L, Lloyd RV (1993) Immunohistochemical analysis of thyroid carcinomas utilizing antibodies to p53 and Ki67. Appl Immunohistochem 1: 201-207
DeLellis R (1995) Does the evaluation of proliferative activity predict malignancy or prognosis in endocrine tumors? Hum Pathol 26: $131-134$

Drobnjak M, Latres E, Pollack D, Karpeh M, Dudas M, Woodruff JM, Brennan MF, Cordon-Cardo C (1994) Prognostic implications of p53 
nuclear overexpression and high proliferation index of KI-67 in adult soft-tissue sarcomas. J Natl Cancer Inst 86: 549-554

Erickson LA, Jin L, Goellner JR, Lohse C, Pankratz VS, Zukerberg LR, Thompson GB, van Heerden JA, Grant CS, Lloyd RV (2000) Pathologic features, proliferative activity, and cyclin D1 expression in Hurthle cell neoplasms of the thyroid. Mod Pathol 13: 186-192

Herfarth KK, Wick MR, Marshall HN, Gartner E, Lum S, Moley JF (1997) Absence of TP53 alterations in pheochromocyomas and medullary thyroid carcinomas. Genes Chromosomes Cancer 20: 24-29

Kerns BJ, Jordan PA, Faerman LL, Berchuck A, Bast Jr RC, Layfield LJ (1994) Determination of proliferation index with MIB-1 in advanced ovarian cancer using quantitative image analysis. Am J Clin Pathol 101: $192-197$

Moley JF, Wells SA, Dilley WG, Tisell LE (1993) Reoperation for recurrent or persistent medullary thyroid cancer. Surgery 114: $1090-1095$

Ozaki O, Ito K, Mimura T, Sugino K (1999) Anaplastic transformation of papillary thyroid. Carcinoma in recurrent disease in regional lymph nodes: a histologic and immunohistochemical study. J Surg Oncol 70: $45-48$

Pence JC, Kerns BJ, Dodge RK, Iglehart JD (1993) Prognostic significance of the proliferation index in surgically resected non-small-cell lung cancer. Arch Surg 128: $1382-1390$

Raymond WA, Leong AS, Bolt JW, Milios J, Jose JS (1988) Growth fractions in human prostatic carcinoma determined by Ki67 immunostaining. J Pathol 156: $161-167$

Rigaud C, Bogomoletz WW (1991) Apparent lack of usefulness of monoclonal antibody Ki-67 in thyroid tumour pathology. Relation to histological typing and classification. Pathol Res Pract 187: 198-200
The Committee on TNM classification UICC (International Union Against Cancer (1992), In TNM Classification of Malignant Tumours, Hermanek P, Sobin LH (eds) pp 35-37. Berlin: Springer Verlag

Tisell LE, Ahlman H, Wängberg B, Hansson G, Mõlne J, Nilsson O, Lindstedt G, Fjälling M, Forssell-Aronsson E (1997) Somatostatin receptor scintigraphy in medullary thyroid carcinoma. $\mathrm{Br} J$ Surg 84: $543-547$

Tisell LE, Dilley WG, Wells SA Jr (1996) Progression of postoperative residual medullary thyroid carcinoma as monitored by plasma calcitonin levels. Surgery 119: 34-39

Tisell LE, Hansson G, Jansson S, Salander H (1986) Reoperation in the treatment of asymptomatic metastasizing medullary carcinoma. Surgery 99: $60-66$

Tubiana M, Courdi A (1989) Cell proliferation kinetics in human solid tumors; relation to probability of metastatic dissemination and longterm survival. Radiother Oncol 15: $1-18$

Wang W, Johansson H, Bergholm U, Wilander E, Grimelius L (1996) Apoptosis and expression of the proto-oncogenes bcl 2 and p53 and the proliferation factor K-67 in human medullary thyroid carcinoma. Endocr Pathol 7: $37-45$

Wells Jr SA, Baylin SB, Linehan WM, Farell RE, Cox EB, Cooper CW (1978) Provocative agents and the diagnosis of medullary carcinoma of the thyroid gland. Ann Surg 188: 139-141

Yana I, Nakamura T, Shin E, Karakawa K, Kurahashi H, Kurita Y, Kobayashi T, Mori T, Nishisho I, Takai S (1992) Inactivation of the p53 gene is not required for tumorigenesis of medullary thyroid carcinoma or pheochromocytoma. Jpn J Cancer Res 83: 1113-1116 